\title{
SLC25A46 Mutations Associated with Autosomal Recessive Cerebellar Ataxia in North African Families
}

\author{
Monia B. Hammer ${ }^{a}$ Jinhui Ding ${ }^{a}$ Fanny Mochel ${ }^{c, d}$ Ghada Eleuch-Fayache ${ }^{f}$ \\ Perrine Charles $^{c}$ Marie Coutelier ${ }^{d}$ J. Raphael Gibbs ${ }^{a}$ Sampath K. Arepalli ${ }^{a}$ \\ Sean B. Chong ${ }^{a}$ Dena G. Hernandez ${ }^{a}$ Elisa Majounie ${ }^{a}$ Steven Clipman $^{a}$ \\ Yosr Bouhlal $^{b}$ Houda Nehdi ${ }^{f} \quad$ Alexis Brice $^{c, d}$ Faycal Hentati ${ }^{f}$ \\ Giovanni Stevanin ${ }^{d, e} \operatorname{Rim}$ Amouri ${ }^{f} \quad$ Alexandra Durr ${ }^{c, d}$ Andrew B. Singleton ${ }^{a}$

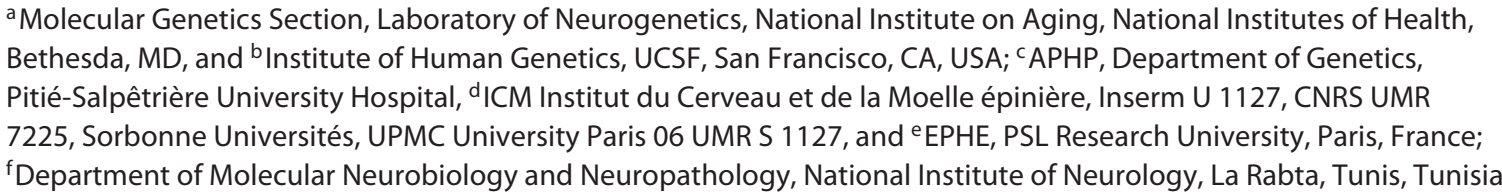

Keywords

Ataxia $\cdot$ SLC25A46 $\cdot$ Mutation · North Africa

\begin{abstract}
Background: Autosomal recessive cerebellar ataxias (ARCA) are a complex group of neurodegenerative disorders with high clinical and genetic heterogeneity. In most cases, the cerebellar ataxia is not pure, and complicating clinical features such as pyramidal signs or extraneurological features are found. Objective: To identify the genetic origin of the cerebellar ataxia for 3 consanguineous North African families presenting with ARCA. Methods: Genome-wide highdensity SNP genotyping and whole-exome sequencing were performed followed by Sanger sequencing for mutation confirmation. Results: Two variants were identified in SLC25A46. Mutations in this gene have been previously associated with Charcot-Marie-Tooth type 2 and optic atrophy. While the previously reported variant p.Arg340Cys seems to
\end{abstract}

\section{KARGER}

(C) 2017 S. Karger AG, Basel

E-Mail karger@karger.com

www.karger.com/ndd be consistently associated with the same clinical features such as childhood onset, optic atrophy, gait and speech difficulties, and wasting of the lower limbs, the patient with the novel mutation p.Trp160Ser did not present with optic atrophy and his ocular abnormalities were limited to nystagmus and saccadic pursuit. Conclusion: In this study, we report a novel variant (p.Trp160Ser) in SLC25A46 and we broaden the phenotypic spectrum associated with mutations in SLC25A46.

(c) 2017 S. Karger AG, Basel

\section{Introduction}

Autosomal recessive cerebellar ataxias (ARCA) are a large group of neurodegenerative disorders that are highly heterogeneous, clinically and genetically. Phenotypic manifestations commonly consist of cerebellar dysarthria, gait ataxia, and dysmetria. Clinical features may be

Andrew B. Singleton

Laboratory of Neurogenetics

NIA, NIH, Building 35, Room 1A1014, 35 Convent Drive

Bethesda, MD 20892 (USA)

E-Mail singleta@ mail.nih.gov 


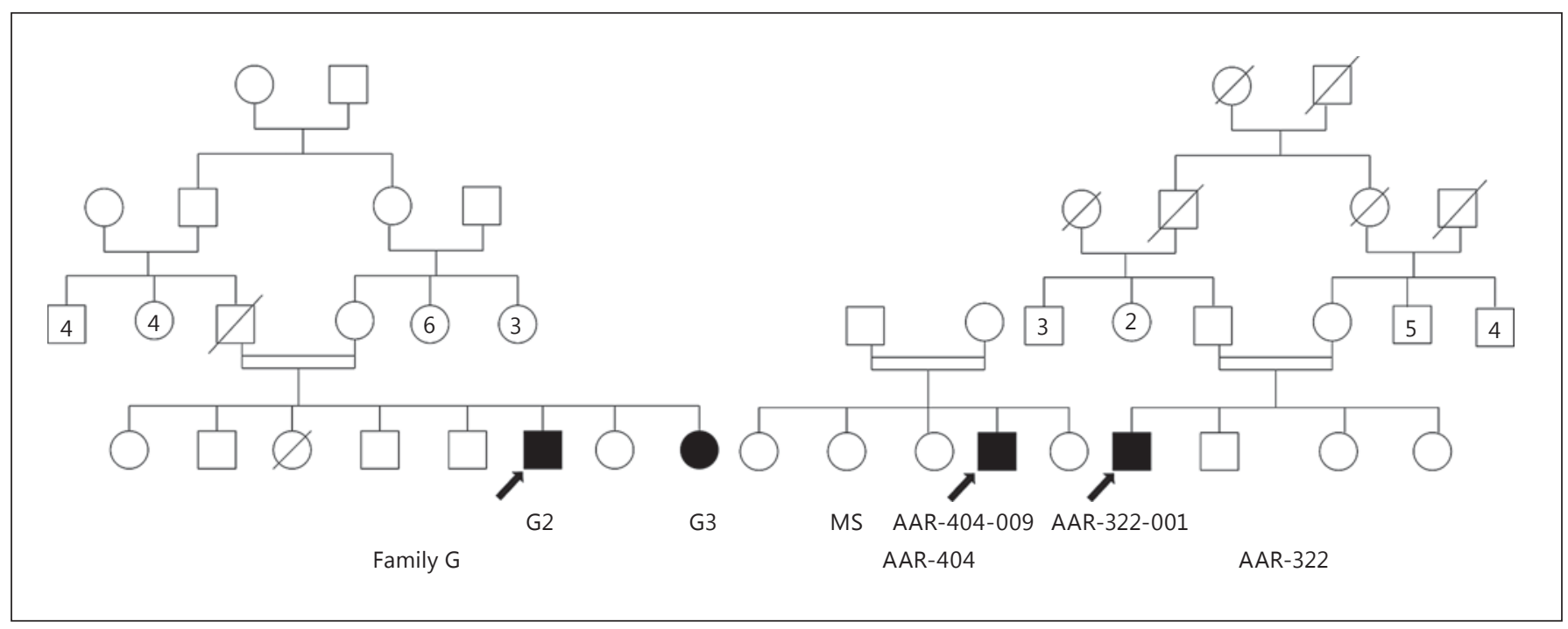

Fig. 1. Family pedigrees. Affected family members are shaded (black). Males are represented by squares, and females are represented by circles.

complicated with gait spasticity, peripheral neuropathy, hearing impairment, or optic atrophy. There are at least 5 forms of ARCA associating cerebellar ataxia with visual impairment: (i) CAMOS (cerebellar ataxia associated with mental retardation, optic atrophy and skin abnormalities), a rare nonprogressive cerebellar ataxia syndrome, originally identified in a Lebanese family [1] and caused by a mutant zinc finger protein, ZNF592 [2]; (ii) IOSCA (infantile onset spinocerebellar ataxia), a severe neurodegenerative disorder that manifests at the age of 9-18 months in previously healthy infants, due to mutations in genes encoding mitochondrial proteins Twinkle and Twinky [3]; (iii) Refsum disease, a hereditary motor sensory neuropathy associated with mutations in $\mathrm{PHYH}$ causing the accumulation of phytanic acid in plasma and lipid-containing tissues [4]; (iv) Boucher-Neuhäuser syndrome, or more broadly the spectrum of PNPLA6-related diseases [5]; and (v) Nyssen-van Bogaert syndrome with a still unknown genetic origin. Recently, Abrams et al. [6] reported 4 mutations in SLC25A46 associated with Charcot-Marie-Tooth type 2 and optic atrophy. In this study, we describe 3 consanguineous North African families with mutations in the same gene but presenting mainly with recessive cerebellar ataxia with or without optic atrophy. The work was conducted at the Laboratory of Neurogenetics, National Institute on Aging, National Institutes of Health, Bethesda, MD, USA.

\section{Methods}

\section{Patients}

Three consanguineous families, 1 of Tunisian descent (family G) and 2 of Algerian descent (families AAR-322 and AAR-404) were studied (pedigrees shown in Fig. 1). All individuals gave informed consent. The work was approved by the local ethics committee and by the Office of Human Subjects Research at the National Institutes of Health.

\section{Molecular Analysis}

Genotyping was performed with the OmniExp-12, v1.0 DNA Analysis BeadChips (Illumina Inc., San Diego, CA, USA) according to the manufacturer's instructions. SNP array data were subjected to homozygosity mapping with HomozygosityMapper software using only homozygous stretches of 15 alleles or longer [7]. Whole-exome sequencing was accomplished according to the Nimblegen v2.0 (Roche Nimblegen, Indianapolis, IN, USA) and the Extended Nextera Rapid-Capture Exome Kit (Illumina) protocols on an Illumina HiSeq 2000. Paired-end sequence reads were aligned against the reference human genome (UCSC hg19). Exome data analysis was accomplished as previously described [8, 9]. Variants were visually inspected with the Integrative Genomics Viewer [10]. Mutation confirmation was done with Sanger sequencing using an ABI BigDye Terminator Cycle Sequencing Kit on an ABI 3730 sequencer. Sequence traces were analyzed via Sequencher version 4.2 (Gene Codes Corporation, Ann Arbor, MI, USA).

Nucleotide and protein positions are based on the following accession numbers from the National Center for Biotechnology Information (http://www.ncbi.nlm.nih.gov/): SLC25A46, NM_ 138773, and NP_620128. Variant positions within the cDNA are numbered using the A of the translation initiation codon as position 1 . 

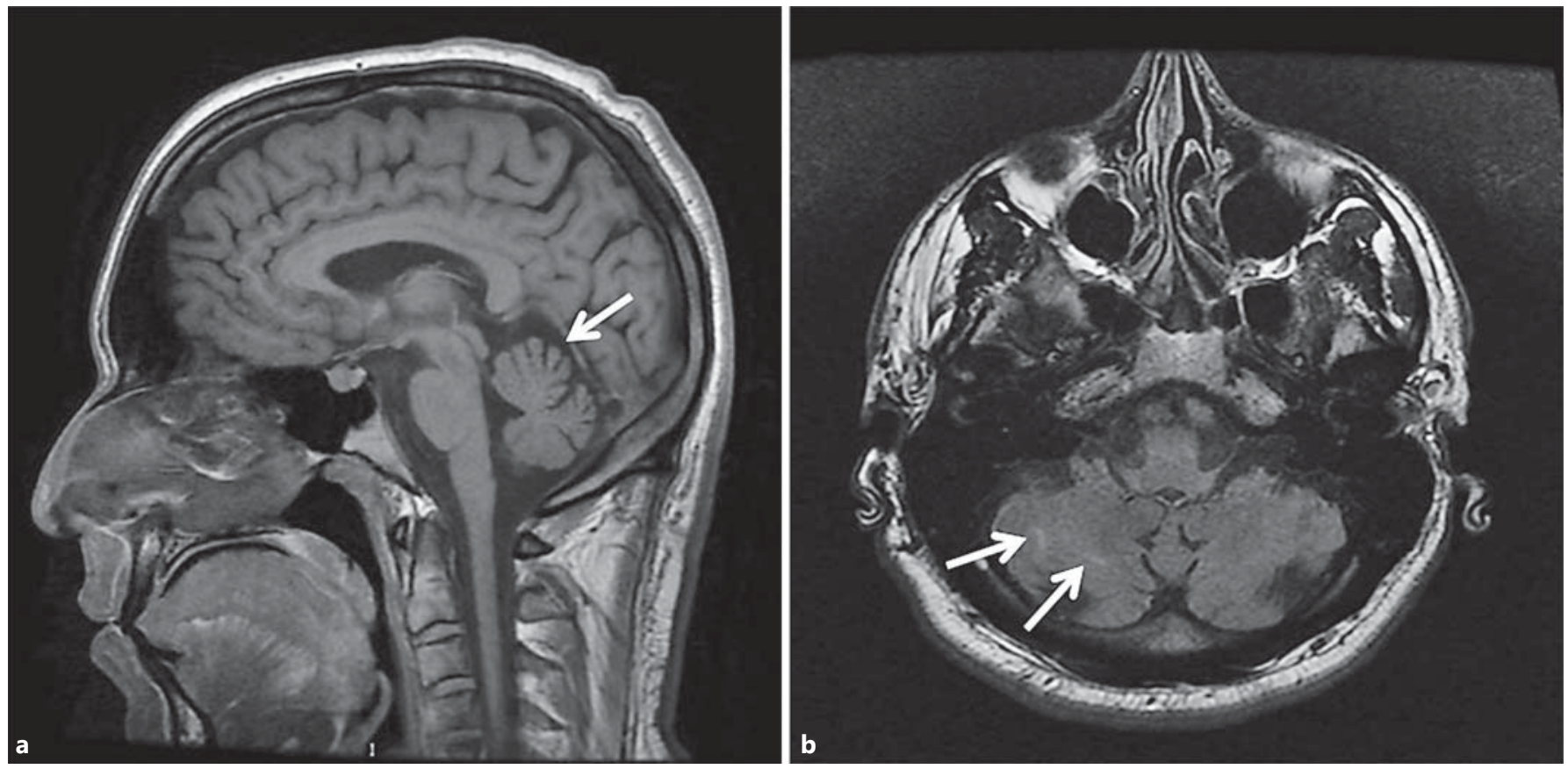

Fig. 2. Brain MRI of patient AAR-322-001 at age 31. Sagittal view of T1-weighted image (a) showing a very mild upper vermis atrophy of the cerebellum (arrow) and an axial FLAIR section (b) showing very subtle white matter abnormalities in the cerebellar hemisphere (arrows).

\section{Results}

\section{Clinical Study}

Family G

We describe 2 siblings (G2 and G3) born from a consanguineous marriage. At the age of 1 year, G2 experienced an outbreak of anterior poliomyelitis causing amyotrophy and shortening of the left lower limb. Later on, he presented with dysarthria and a decrease in visual acuity that forced him to quit school by the age of 10 . At age 14 , the patient showed gait instability. At 22, he was wheelchair-bound. Neurological examination revealed bilateral plantar extensor responses, abolished Achilles reflexes, and finger to nose dysmetria. Nystagmus, divergent strabismus (right eye), blindness, and bilateral optic nerve atrophy were found at the eye examination. Cerebral tomodensitometry showed a cerebellar mega cisterna without atrophy. A nerve conduction study revealed severe sensorimotor demyelinating neuropathy. Nerve biopsy exhibited hypertrophic neuropathy with segmental demyelination, giant axons, and onion bulb formations. His sister G3 presented at age 6 with a decrease in visual acuity, especially on the left side. At age 8 , she showed walking difficulties. At 19, walking became im- possible. Neurological examination revealed mild dysarthria, lower limb wasting, a Babinski sign, finger to nose dysmetria, and abolished knee and Achilles reflexes. Skeletal abnormalities such as scoliosis and hollow feet were found. The eye examination revealed bilateral divergent strabismus, a decrease in visual acuity, and optic atrophy in both eyes. A nerve conduction study showed severe sensorimotor axonal neuropathy, predominantly in the lower limbs. Superficial peroneal nerve biopsy indicated the presence of onion fibers and a decrease in myelinated fibers.

\section{Patient AAR-322-001}

The patient was born from a consanguineous union. He was diagnosed with bilateral optic atrophy at 2 years of age. He started developing gait difficulties when he was 17 years old, with an ataxic gait due to sensory deficit. Distal motor deficit and amyotrophy in his lower limbs led to the diagnosis of sensorimotor axonal polyneuropathy. The patient also presented with mild pyramidal features with increased tendon reflexes, except for the Achilles reflexes that were absent, a positive Hoffmann sign, but no Babinski sign. Muscle biopsy showed abnormal subsarcolemnic accumulations without typical mito- 
Fig. 3. SLC25A46 domains and functional sites. TM, transmembrane.

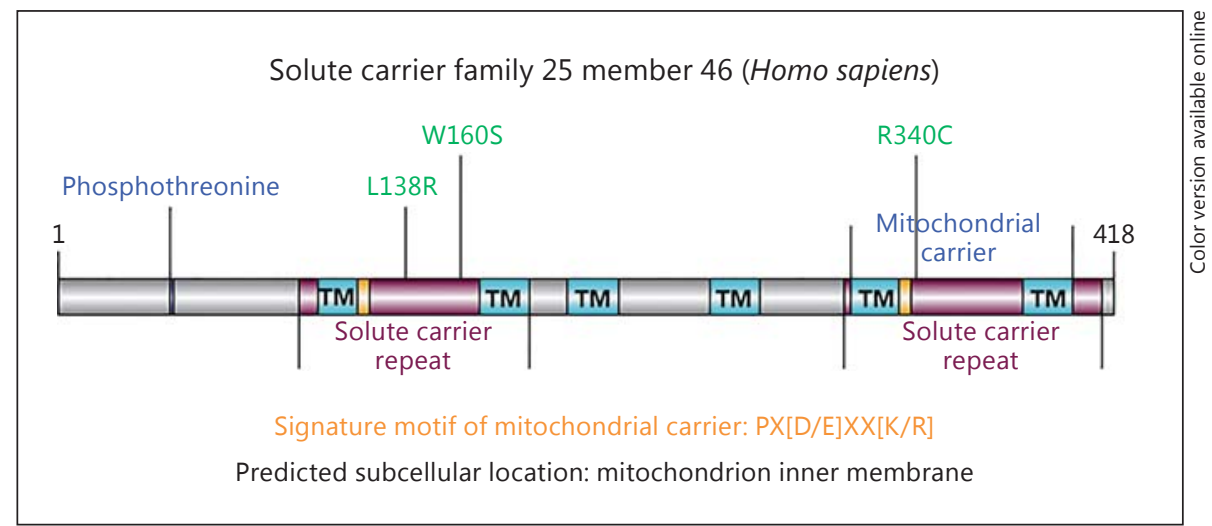

chondrial features. Subtle white matter changes in the cerebellum were noticed on cerebral MRI at age 31 (Fig. 2). Other clinical features included arrhythmia and dyspnea.

\section{Patient AAR-404-009}

This patient also originates from a consanguineous family with first cousin parents. He presented with gait unsteadiness at age 23 and a complaint of "not feeling the ground." At age 26, examination revealed moderate gait ataxia and mild lower limb ataxia, with a disability stage of 2 (mild, able to run). Plantar reflexes were indifferent. No motor deficit was noted. Vibration sense at ankles was abolished. The patient had mild scoliosis and presented with nystagmus and saccadic pursuit. EMG revealed axonal peripheral neuropathy. Alpha-fetoprotein, cholester$\mathrm{ol}$, and vitamin $\mathrm{E}$ were normal. An older sister of the proband had a clinical diagnosis of multiple sclerosis.

\section{Molecular Study}

Family G was initially studied. Genome-wide genotyping of both affected individuals and their mother was conducted. Homozygosity mapping revealed a total of 15 homozygous regions across 9 different chromosomes. The exome data showed that, within these regions, only 2 variants were shared between the patients. The first region located on chromosome 1 (chr1: 235,523,555-241,183, 893 ) includes a variant (T131S) in NID1. The second region, on chromosome 5 spanning from rs4704382 (chr5: $76,318,206 \mathrm{bp}$ ) to rs10149476 (chr5: $107,287,663 \mathrm{bp}$ ), comprises a substitution of arginine for cysteine at residue 340 (c.1018C>T, p.Arg340Cys) in SLC25A46. While this study was ongoing, Abrams et al. [6] reported the same variant in a Sardinian family. Sanger sequencing confirmed that the variant was homozygous in both sib-

SLC25A46 Mutations in ARCA lings and heterozygous in the mother. Subsequently, we looked for mutations in SLC25A46 in our exome data of similar cases and identified the same variant, p.Arg$340 \mathrm{Cys}$, and a potentially novel variant in 2 unrelated Algerian families. The novel variant consists of a homozygous substitution c. $479 \mathrm{G}>\mathrm{C}$ that changes the tryptophan residue at position 160 to a serine (p.Trp160Ser). Public database searches (dbSNP138, NHLBI ESP6500, and ExAC) revealed that this variant was not reported. It is predicted to be damaging by Polyphen $2($ score $=1)$, SIFT $($ score $=0)$ and MutationTaster (score $=1)$. Moreover, tryptophan 160 is a highly conserved amino acid residue across species.

\section{Discussion}

By sequence similarity analysis, SLC25A46 encodes a mitochondrial carrier protein. It is located in the mitochondrion inner membrane and is a multipass membrane protein. As are many other members in the mitochondrial carrier family $[11,12]$, SLC25A46 is predicted to consist of 6 transmembrane alpha-helices, TM1-TM6 (Fig. 3). Both mutations, p.Arg340Cys and p.Trp160Ser, are on the loops of the repeat sequence facing the inside matrix space. p.Trp160Ser is on the matrix loop between transmembrane helix TM1 and TM2, while p.Arg340Cys is on the matrix loop between TM5 and TM6. The arginine at position 340 is a highly conserved residue in the mitochondrial carrier family (NCBI Conserved Domains Database), and it is adjacent to the highly conserved signature motif $\mathrm{PX}[\mathrm{D} / \mathrm{E}] \mathrm{XX}[\mathrm{K} / \mathrm{R}]$, which is characteristic of all mitochondrial carriers. It is pointing to the mitochondria matrix space. The position-specific scoring matrix (PSSM) score (log-odds score, basically calculated as the

Neurodegener Dis 2017;17:208-212 211 
$\log$ (base 2) of the observed substitution frequency at a given position divided by the expected substitution frequency at that position) for p.Arg340Cys is -9 , suggesting that the substitution is pathogenic, especially in structures of the mitochondrial carrier protein family. p.Trp160 Ser is a change from nonpolar to polar decreasing hydrophobicity and is pathogenic based upon the PSSM scores. Slc25a46 is important for both the growth and maintenance of neuronal processes, and these mutations may affect the normal function of the protein.

Abrams et al. [6] reported a wide phenotypic range associated with mutations in SLC25A46 from mild, with patients well and fit until their 40 s, to lethal. In fact, different mutations seem to trigger different phenotypes. The p.Arg340Cys mutation seems to be consistently associated with the same clinical features across families (the Tunisian and Algerian families reported in this study and the Sardinian family from Abrams' paper) with childhood onset, optic atrophy, cerebellar or sensitive ataxia, speech difficulties, and wasting of the lower limbs due to polyneuropathy. The clinical description associated with the p.Trp160Ser mutation surprisingly lacked the presence of optic atrophy, and ocular abnormalities were limited to nystagmus and saccadic pursuit. Also, the age at onset was later in life (23 years of age) in comparison with all of the other families (under 15 years of age). Ataxia was observed in 2 families (Palestinian and Sardinian) out of 4 reported by Abrams et al. [6], while it was the striking feature in our patients.

In this study, we broadened the phenotypic spectrum associated with mutations in SLC25A46. This gene seems to be linked to neurological disorders worldwide.

\section{Acknowledgments}

The authors thank J. Hammer for his contribution in the correction of the manuscript and his help with the figures. This work was supported in part by the Intramural Research Programs of the National Institute on Aging and the National Institutes of Neurological Disorders and Stroke (within the National Institutes of Health, Department of Health and Human Services: project number ZO1 AG000958), and the National Ataxia Foundation. We are also grateful to the European Union (7th PCRD, Omis Call, grant NEUROMICS), the VERUM foundation, and Connaitre Les Syndromes Cérébelleux.

\section{Disclosure Statement}

The authors declare no conflicts of interest.

\section{References}

1 Mégarbané A, Delague V, Ruchoux MM, Rizkallah E, Maurage CA, Viollet L, Rouaix-Emery N, Urtizberea A: A new autosomal recessive cerebellar ataxia disorder in a large inbred Lebanese family. Am J Med Genet 2001;101: 135-141.

2 Nicolas E, Poitelon Y, Chouery E, Salem N, Levy N, Mégarbané A, Delague V: CAMOS, a nonprogressive, autosomal recessive, congenital cerebellar ataxia, is caused by a mutant zinc-finger protein, ZNF592. Eur J Hum Genet 2010;18:1107-1113.

3 Nikali K, Suomalainen A, Saharinen J, Kuokkanen M, Spelbrink JN, Lönnqvist T, Peltonen $\mathrm{L}$ : Infantile onset spinocerebellar ataxia is caused by recessive mutations in mitochondrial proteins Twinkle and Twinky. Hum Mol Genet 2005; 14:2981-2990.

4 Wierzbicki AS, Lloyd MD, Schofield CJ, Feher MD, Gibberd FB: Refsum's disease: a peroxisomal disorder affecting phytanic acid alpha-oxidation. J Neurochem 2002;80:727735.
5 Synofzik M, Gonzalez MA, Lourenco CM, Coutelier M, Haack TB, Rebelo A, Hannequin D, Strom TM, Prokisch H, Kernstock C, Durr A, Schöls L, Lima-Martínez MM, Farooq A, Schüle R, Stevanin G, Marques W Jr, Züchner S: PNPLA6 mutations cause BoucherNeuhäuser and Gordon Holmes syndromes as part of a broad neurodegenerative spectrum. Brain 2014;137(Pt 1):69-77.

6 Abrams AJ, Hufnagel RB, Rebelo A, Zanna C, Patel N, Gonzalez MA, et al: Mutations in SLC25A46, encoding a UGO1-like protein, cause an optic atrophy spectrum disorder. Nat Genet 2015;47:926-932.

7 Li H, Durbin R: Fast and accurate short read alignment with Burrows-Wheeler transform. Bioinformatics 2009;25:1754-1760.

8 Hammer MB, Eleuch-Fayache G, Gibbs JR, Arepalli SK, Chong SB, Sassi C, Bouhlal Y, Hentati F, Amouri R, Singleton AB: Exome sequencing: an efficient diagnostic tool for complex neurodegenerative disorders. Eur J Neurol 2013;20:486-492.
9 Hammer MB, Eleuch-Fayache G, Schottlaender LV, Nehdi H, Gibbs JR, Arepalli SK, Chong SB, Hernandez DG, Sailer A, Liu G, Mistry PK, Cai H, Shrader G, Sassi C, Bouhlal Y, Houlden H, Hentati F, Amouri R, Singleton AB: Mutations in GBA2 cause autosomalrecessive cerebellar ataxia with spasticity. Am J Hum Genet 2013;92:245-251.

10 Robinson JT, Thorvaldsdottir H, Winckler W, Guttman M, Lander ES, Getz G, Mesirov JP: Integrative genomics viewer. Nat Biotechnol 2011;29:24-26.

11 Pebay-Peyroula E, Dahout-Gonzalez C, Kahn R, Trézéguet V, Lauquin GJ, Brandolin G: Structure of mitochondrial ADP/ATP carrier in complex with carboxyatractyloside. Nature 2003;426:39-44.

12 Berardi MJ, Shih WM, Harrison SC, Chou JJ: Mitochondrial uncoupling protein 2 structure determined by NMR molecular fragment searching. Nature 2011;476:109-113. 